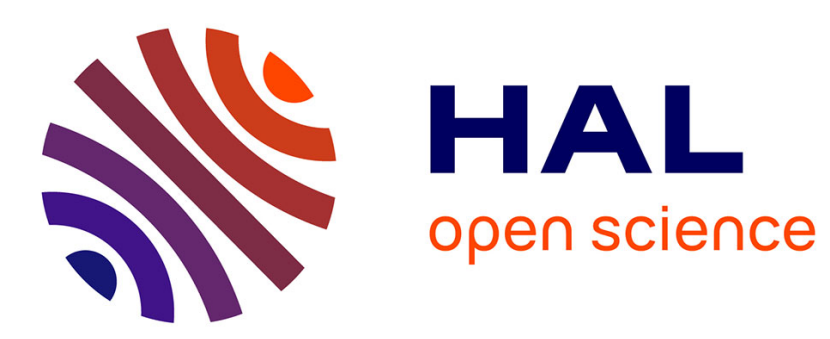

\title{
Kalman Filter Based Observer for an External Force Applied to Medium-sized Humanoid Robots
}

\author{
Louis Hawley, Rémy Rahem, Wael Suleiman
}

\section{To cite this version:}

Louis Hawley, Rémy Rahem, Wael Suleiman. Kalman Filter Based Observer for an External Force Applied to Medium-sized Humanoid Robots. IEEE/RSJ International Conference on Intelligent Robots and Systems, Oct 2018, Madrid, Spain. hal-01862107

\section{HAL Id: hal-01862107 \\ https://hal.science/hal-01862107}

Submitted on 27 Aug 2018

HAL is a multi-disciplinary open access archive for the deposit and dissemination of scientific research documents, whether they are published or not. The documents may come from teaching and research institutions in France or abroad, or from public or private research centers.
L'archive ouverte pluridisciplinaire HAL, est destinée au dépôt et à la diffusion de documents scientifiques de niveau recherche, publiés ou non, émanant des établissements d'enseignement et de recherche français ou étrangers, des laboratoires publics ou privés. 


\title{
Kalman Filter Based Observer for an External Force Applied to Medium-sized Humanoid Robots
}

\author{
Louis Hawley, Rémy Rahem and Wael Suleiman
}

\begin{abstract}
External force observer for humanoid robots has been widely studied in the literature. However, most of the proposed approaches generally rely on information from sixaxis force/torque sensors, which the small or medium-sized humanoid robots usually do not have. As a result, those approaches cannot be applied to this category of humanoid robots, which is widely used nowadays in education or research.

In this paper, we improve the external force observer in [1] to handle the case of an external force applied in any direction and at an arbitrary point of the robot structure. The new observer is based on Kalman filter formulation and it allows the estimation of the three force components. The observer is simple to implement and can easily run in real time using the embedded processor of a medium-sized humanoid robot such as Nao or Darwin-OP. Moreover, the observer does not require any change to the robot hardware as it only uses measurements from the available force-sensing resistors (FSR) inserted under the feet of the humanoid robot and from the robot inertial measurement unit (IMU).

The proposed observer was extensively validated on a Nao humanoid robot. In all conducted experiments, the observer successfully estimated the external force within a reasonable margin of error.
\end{abstract}

\section{INTRODUCTION}

Having a biped structure and articulated arms makes humanoid robots ideal candidates to perform a variety of manipulation and transportation tasks. However, the manipulation or transportation of an object may create instability for the humanoid robot if no measures are taken to consider the added force, which is generated by the transported object, and adjust the walking gait. Moreover, physical interaction with a human, such as collaboratively transporting an object, also generates interaction force that should be taken into account to prevent instability issues. In those cases, it is primordial to accurately estimate the force acting on the humanoid robot.

As an example, the transportation of a heavy object on a rolling cart, as depicted in [2], [3], can be greatly improved if the external force generated by the cart and the transported object can be accurately measured or estimated. In [3], a planning and navigation framework for the transportation of an object on a rolling cart have been proposed. The framework generates a feasible trajectory for the humanoid robot and the cart while considering the physical limitations of the robot. In the proposed implementation, the robot is only able to differentiate between light and heavy loads by executing a turning in place motion with the cart, and it

Authors are with Electrical and Computer Engineering Department, the Faculty of Engineering, University of Sherbrooke, Quebec, Canada LLouis.Hawley, Remy.Rahem, Wael.Suleiman\}@USherbrooke.ca

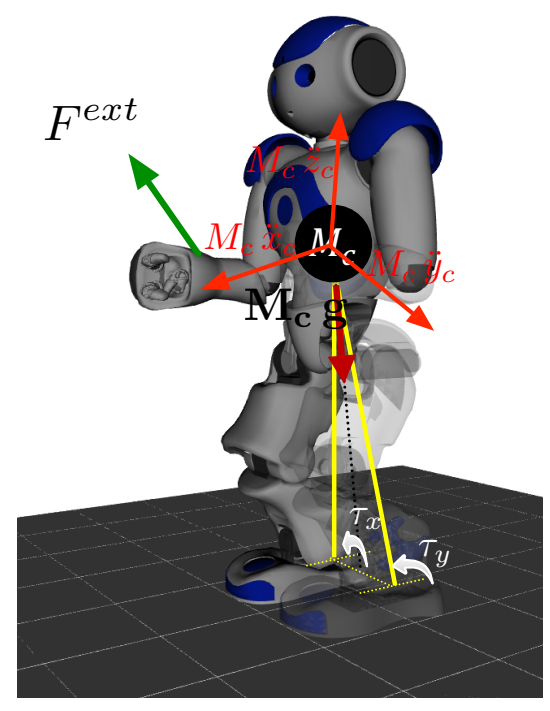

Fig. 1. An external force applied to a Nao robot

then uses the appropriate set of motion primitives in the planning phase. However, it would be useful to have a better estimation of the external force exerted on the humanoid robot for: I)- an optimal motion planning, II)- more stable locomotion by integrating the external force into the pattern generation module.

Moreover, for a cooperative transportation task of an object with two humanoid robots, as presented in [4], [5], it would be very useful to measure the force exchanged through the object in order to maintain the equilibrium of the whole system.

\section{A. Relevant Works}

There are multiple approaches to estimate external forces exerted on a humanoid robot. These approaches can be roughly separated into three main categories : sensor-based approaches, model-based approaches and alternative methods such as machine learning-based methods. Each category is summarized with some examples in the following subsections.

1) Sensor-based Approach: The sensor-based approach directly measures the external force applied to a humanoid robot by using the robot's sensors. Such a method was used in [6], [7], where a pushing manipulation task was performed by a HRP- 2 robot. In that case, the external force applied to the humanoid robot can be directly measured at all time 
using the six-axis Force/Torque (F/T) sensors embedded in the robot's wrists. Then, by considering the external force, the authors were able to apply the required compensation on the torso position to ensure the stability of the robot while pushing the object.

A similar approach has also been proposed by the same authors to enable a humanoid robot to lift and transport a heavy object [8]. The sensor-based approach obviously provides great performance as the force can be accurately measured at all times. However, F/T sensors are not available on all robots and it would be difficult to integrate them on smaller and cheaper robots due to the size and cost of such sensors.

2) Model-based Approach: We define model-based approach as the methods that estimate the external force acting on a humanoid robot by using a dynamic model of the robot and its actual state vector. For instance, the method presented in [9], where the magnitude of an external force is estimated using a disturbance observer. The observer uses measurements from the robot's IMU and from F/T sensors located in the ankles to estimate the magnitude of a strong force such as a kick to the robot chest. In order to estimate the disturbance, the authors used a simplified dynamic model of the humanoid where the acceleration of its Center of Mass (CoM) is a function of the forces applied to the robot, which are: the gravity, the floor reaction force and the unknown external forces. Since the acceleration of the robot's CoM is known using IMU measurements and the floor reaction force can be directly measured with the F/T sensors, their results show that a strong impact force can be estimated somewhat accurately using that model. Another interesting method that can also estimate the external moment is proposed in [10], that method relies on a model-based estimator to fuse the data of the robot's sensors (F/T sensors and IMU) and the whole body dynamics. An advantage of this approach over the previously presented sensor-based approach is that the external force/moment do not have to be applied at a particular point in order to be estimated. However, these methods still require F/T sensors.

A similar approach has been presented in [11] to enable a humanoid robot to walk on low friction floor. Using the linear inverted pendulum model (LIPM) to represent the humanoid robot, the authors predicted the ground reaction force and compared it with the measurements from F/T sensors in the ankles of a HRP-2 robot. The difference between the two values was then attributed to a slip force that can be easily derived. Although a slip force is also a strong force of short duration, which is very different from the force encountered in interaction tasks between several robots or a robot and a human, these works are interesting as they show that it is possible to get a good estimation of an external force by only using a limited number of sensors and a simple dynamic model of the robot. Such an approach would be interesting for medium-sized humanoid, such as Nao humanoid robot, as they usually have a small number of sensors and limited computational power. Nao [12] is a medium-sized humanoid robot manufactured by SoftBank Robotics.
In [13], the Nao robot's limbs are modeled as kinematic chains attached to the pelvis of the robot, and a relation between a force acting on each link of the robot and the joint torques is established, a method commonly used for serial manipulators. Since Nao's joints do not possess torque sensors nor do they provide a torque estimation, the authors had to define an actuator model to estimate the motor torque from the steady-state error. Then, the problem is transformed into isolating the torque caused by gravity to obtain the portion of the torque that is the result of the external force. Although this approach can be quite powerful as the magnitude of the force along with the contact point can be found, there are multiple drawbacks in the proposed implementation. For instance, it uses the motors steadystate error to estimate the actuator torque. That means the proposed algorithm is mainly able to estimate quite a big external force.

Rotella et al. [14] presented some formulations to estimate the CoM position of a robot and to correct inaccuracies in the robot links model. With regard to our work, the most interesting contribution is an external wrench estimator that uses a linear and angular momentum model of the robot instead of the simplified LIPM dynamic. However, the approach is limited to humanoids equipped with F/T sensors at the end-effectors.

Another model-based method to estimate the magnitude of an external force applied to a humanoid robot is presented in [1]. Its main advantage is that it does not require using $\mathrm{F} / \mathrm{T}$ sensors but instead it uses measurements from the robot's force-sensing resistors (FSR) sensors. The approach has been successfully validated on a Nao robot and pointed out that an external force applied to the robot can be efficiently estimated with an acceptable precision. However, the main limitation of that method is that the formulations are only valid for an external force applied in the horizontal plane.

3) Alternative Methods: Machine learning based approaches have also proven to be useful in estimating an external force applied to medium-sized/small humanoid robots.

In [15], perturbations transmitted to a Nao robot are detected by comparing the actual sensor measurements with the predicted ones obtained with a probabilistic model. Using this method, the authors were able to successfully detect and provide an estimation of the perturbation magnitude. However, the external force that generates the perturbation has never been quantified as the algorithm can either provide the difference between the sensors (IMU and FSR) measurements and the predicted values or an equivalent footstep length. Although it is certainly possible to formulate an estimation of the force from these results, such analysis has not been conducted.

In [16], the authors present a complimentary filter formulation to estimate the CoM of a robot or human. The proposed filter uses a spectral approach to analyze the different methods used to estimate the position of CoM (i.e. motion capture, contact forces and moments). Then, the authors propose to fuse the data by optimally using each sensor. Obviously, this approach requires a diverse set of 
sensors to span the whole frequency range, a condition that is often not satisfied in the case of small humanoid robots.

The main contribution of this paper is the design of a model-based observer that is able to estimate the three components of an external force applied to a humanoid robot. The force is projected onto the CoM frame, and therefore it can be directly used, for instance, in a pattern generator. The observer design and implementation architecture are given in Section IV.

This observer was also validated on a Nao humanoid robot through several experimental scenarios. The results of these experiments are analyzed in Section VI.

\section{ZMP WITH AN EXTERNAL FORCE}

To study the impact of an external force on the ZeroMoment Point (ZMP) formulation, we consider the two following cases:

\section{A. An External Force Directly Applied to the Robot CoM}

This case study is illustrated in Fig. 2. The ZMP can be found by considering the forces acting on the simplified LIPM model, which are the gravity, the inertial force and the external force.

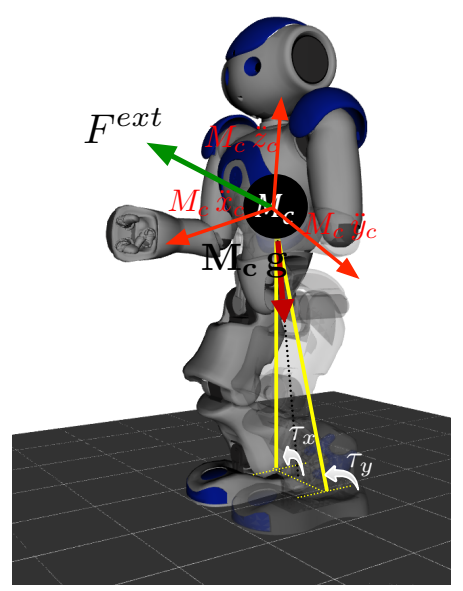

Fig. 2. An external external force applied to the CoM of a Nao robot

Let the ZMP on the horizontal ground be given by the following vector ${ }^{1}$ :

$$
\boldsymbol{p}=\left[\begin{array}{ll}
p_{x} & p_{y}
\end{array}\right]^{T}
$$

where $p_{x}$ and $p_{y}$ are respectively the projections of the ZMP on the $x$ and $y$ axis.

To compute $\boldsymbol{p}$, one can use the following formula:

$$
\boldsymbol{p}=\boldsymbol{N} \frac{\boldsymbol{n} \times \boldsymbol{\tau}^{o}}{\left(\boldsymbol{f}^{\circ} \mid \boldsymbol{n}\right)}
$$

where the operators $\times$ and (.|.) design respectively the cross and scalar products, and

\footnotetext{
${ }^{1}$ As a general rule, bold parameters are vectors or matrices
}

- $N$ is a constant matrix

$$
\boldsymbol{N}=\left[\begin{array}{lll}
1 & 0 & 0 \\
0 & 1 & 0
\end{array}\right]
$$

- the vector $\boldsymbol{n}$ is the normal vector on the horizontal ground $\left(\boldsymbol{n}=\left[\begin{array}{lll}0 & 0 & 1\end{array}\right]^{T}\right)$.

- The vector $f^{o}$ is the result of the gravity, inertial and external force:

$$
\boldsymbol{f}^{o}=M_{c} \boldsymbol{g}-M_{c} \ddot{\boldsymbol{X}}_{c}+\boldsymbol{F}^{e x t}
$$

where $\boldsymbol{g}$ denotes the gravity acceleration $(\boldsymbol{g}=-g \boldsymbol{n})$, $M_{c}$ is the total mass of the humanoid robot, and $\ddot{\boldsymbol{X}}_{c}=\left[\begin{array}{lll}\ddot{x}_{c} & \ddot{y}_{c} & \ddot{z}_{c}\end{array}\right]^{T}$ is the acceleration of the CoM. $\boldsymbol{F}^{e x t}=\left[\begin{array}{lll}F_{x} & F_{y} & F_{z}\end{array}\right]^{T}$ is the external force applied to the humanoid robot.

The normal force is given by the following expression:

$$
\left(\boldsymbol{f}^{o} \mid \boldsymbol{n}\right)=f_{n}^{o}=-M_{c} g-M_{c} \ddot{z}_{c}+F_{z}
$$

- $\tau^{o}$ denotes the moment of the external force and the moment of the force $f^{o}$ about the origin of the fixed world frame, it is given by the following expression:

$$
\boldsymbol{\tau}^{o}=\boldsymbol{X}_{c} \times \boldsymbol{f}^{o}
$$

where $\boldsymbol{X}_{c}=\left[\begin{array}{lll}x_{c} & y_{c} & z_{c}\end{array}\right]^{T}$ is the Cartesian position of CoM.

Thus, we obtain:

$$
\boldsymbol{p}=\left[\begin{array}{l}
p_{x} \\
p_{y}
\end{array}\right]=\left[\begin{array}{l}
x_{c}+\frac{M_{c} z_{c}}{f_{n}^{o}} \ddot{x}_{c}-\frac{z_{c}}{f_{n}^{o}} F_{x} \\
y_{c}+\frac{M_{c} z_{c}}{f_{n}^{o}} \ddot{y}_{c}-\frac{z_{c}}{f_{n}^{o}} F_{y}
\end{array}\right]
$$

\section{B. An External Force Applied to a Point of the Robot}

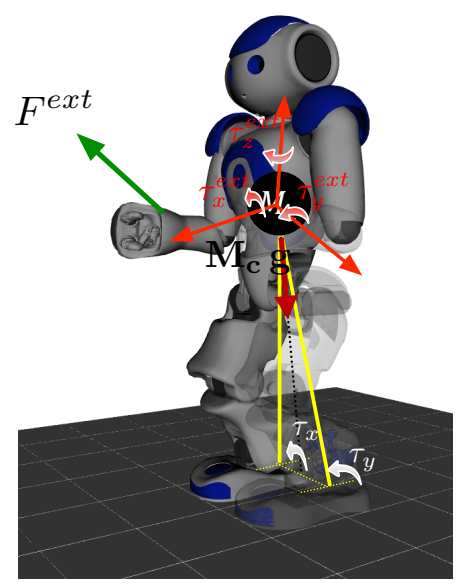

Fig. 3. An external moment is applied to the CoM of LIPM model as a result of an external force applied to a point of Nao robot, the accelerations are omitted for clarity

An external force applied to a point of the robot structure generates an additional external moment applied to the CoM of LIPM model as shown in Fig. 3. For instance, this force 
could be the result of the robot pushing, pulling or lifting an object or interacting/collaborating with a human.

Similarly to the previous case, the resultant force applied to the LIPM model is:

$$
\boldsymbol{f}^{o}=M_{c} \boldsymbol{g}-M_{c} \ddot{\boldsymbol{X}}_{c}+\boldsymbol{F}^{e x t}
$$

However, the moment $\boldsymbol{\tau}^{o}$ in this case becomes:

$$
\boldsymbol{\tau}^{o}=\boldsymbol{X}_{c} \times \boldsymbol{f}^{o}+\boldsymbol{\tau}^{e x t}
$$

By applying the formula in (2), we obtain:

$$
\begin{aligned}
\boldsymbol{p} & =\left[\begin{array}{l}
p_{x} \\
p_{y}
\end{array}\right] \\
& =\left[\begin{array}{l}
x_{c}+\frac{M_{c} z_{c}}{f_{n}^{o}} \ddot{x}_{c}-\frac{z_{c}}{f_{n}^{o}} F_{x}-\frac{1}{f_{n}^{o}} \tau_{y}^{e x t} \\
y_{c}+\frac{M_{c} z_{c}}{f_{n}^{o}} \ddot{y}_{c}-\frac{z_{c}}{f_{n}^{o}} F_{y}+\frac{1}{f_{n}^{o}} \tau_{x}^{e x t}
\end{array}\right] \\
& =\left[\begin{array}{l}
x_{c}+\frac{M_{c} z_{c}}{f_{n}^{o}} \ddot{x}_{c}-\frac{z_{c}}{f_{n}^{o}}\left(F_{x}+\frac{\tau_{y}^{e x t}}{z_{c}}\right) \\
y_{c}+\frac{M_{c} z_{c}}{f_{n}^{o}} \ddot{y}_{c}-\frac{z_{c}}{f_{n}^{o}}\left(F_{y}-\frac{\tau_{x}^{e x t}}{z_{c}}\right)
\end{array}\right]
\end{aligned}
$$

It is clear from (10) that, without additional sensors such as a six-axis F/T sensor, the components of external force and moment cannot be estimated. However, by defining the following virtual force:

$$
\begin{aligned}
\hat{\boldsymbol{F}}^{e x t} & =\left[\begin{array}{c}
\hat{F}_{x} \\
\hat{F}_{y} \\
\hat{F}_{z}
\end{array}\right] \\
& \triangleq\left[\begin{array}{c}
F_{x}+\frac{\tau_{y}^{e x t}}{z_{c}} \\
F_{y}-\frac{\tau_{x}^{\text {ext }}}{z_{c}} \\
F_{z}
\end{array}\right]
\end{aligned}
$$

One can figure out that, from ZMP point of view, the impact of an external force $\boldsymbol{F}^{e x t}$ applied to a point of the robot is the same as a virtual force $\hat{\boldsymbol{F}}^{\text {ext }}$ applied directly to the CoM of LIPM model. Therefore, we only consider the latter case in the sequel of the paper.

\section{MEASUREMENTS}

Using the robot available sensors (FSR, IMU and joint encoders), the ZMP, CoM position and acceleration, and ground reaction force can be estimated. Those variables will play the role of output (observation) signals for the Kalman filter based observer. Note that, in this work, all measurements are unfiltered (raw) signals.

\section{A. Ground Reaction Force}

The ground reaction force can be directly estimated using the FSR sensors. In the case of Nao robot, for instance, each foot possesses $4 \mathrm{FSR}$ sensors with a force range between 0 and $25 \mathrm{~N}$, and the ground reaction force $f_{n}^{o}$ is given by:

$$
f_{n}^{o}=-(\underbrace{\sum_{i=1}^{4} \mathcal{F}_{i}^{R}}_{\text {right foot }}+\underbrace{\sum_{i=1}^{4} \mathcal{F}_{i}^{L}}_{\text {left foot }})
$$

where $\mathcal{F}_{i}^{*}$ is the force measured at the $i^{t h} \mathrm{FSR}$.

\section{B. $Z M P$}

Recall that the ZMP and Center of Pressure $(\mathrm{CoP})$ coincide when the robot is in a stable configuration [17]. Thus, the ZMP can be computed as follows:

$$
\begin{gathered}
p_{x}=x_{C o P}=\frac{\sum_{i=1}^{4} \mathcal{F}_{i}^{R} x_{i}^{R}+\sum_{i=1}^{4} \mathcal{F}_{i}^{L} x_{i}^{L}}{\left|f_{n}^{o}\right|} \\
p_{y}=y_{C o P}=\frac{\sum_{i=1}^{4} \mathcal{F}_{i}^{R} y_{i}^{R}+\sum_{i=1}^{4} \mathcal{F}_{i}^{L} y_{i}^{L}}{\left|f_{n}^{o}\right|}
\end{gathered}
$$

where $f_{n}^{o}$ is defined in (12), and $x_{i}^{*}$ and $y_{i}^{*}$ are the $x$ and $y$ positions of the $i^{t h}$ FSR in the foot frame.

\section{CoM Acceleration}

The acceleration of CoM can be directly extracted from the IMU, which is a standard part of a humanoid robot sensors. As for Nao, the IMU provides measurements from a three-axis accelerometer and a two-axis gyroscope. As in [1], we assume that the CoM coincides with the IMU. Therefore, the accelerometer data can be used to approximate the acceleration of the CoM.

\section{CoM Position}

The CoM position in the support foot frame can be easily obtained by a simple forward kinematics.

\section{EXTERNAL ForCE OBSERVER}

The first component of the external force applied to the humanoid robot that can be directly estimated from the FSR information is $F_{z}$ using (5). Then, the horizontal components $F_{x}$ and $F_{y}$ can be estimated using (7).

\section{A. Estimating $F_{z}$}

To estimate $F_{z}$, Eq. (5) is discretized with a sampling time $T$ to obtain the following linear dynamic system:

$$
\begin{aligned}
\mathcal{Z}(k+1) & =\boldsymbol{A} \mathcal{Z}(k)+\boldsymbol{B} \boldsymbol{u}_{z}(k) \\
\boldsymbol{Y}_{z}(k) & =\boldsymbol{C}_{z} \mathcal{Z}(k)+\boldsymbol{v}_{z}(k)
\end{aligned}
$$

where:

- The state vector:

$$
\mathcal{Z}(k)=\left[\begin{array}{lllll}
z_{c}(k T) & \dot{z}_{c}(k T) & \ddot{z}_{c}(k T) & F_{z}(k T) & \dot{F}_{z}(k T)
\end{array}\right]^{T}
$$

- The input signal:

$$
\boldsymbol{u}_{z}(k)=\left[\begin{array}{ll}
\dddot{z}_{c}(k T) & \ddot{F}_{z}(k T)
\end{array}\right]^{T}
$$

where $\dddot{z}_{c}(k T)$ is the acceleration derivative which is usually called the jerk.

- The output signal:

$$
\boldsymbol{Y}_{z}(k)=\left[\begin{array}{lll}
z_{c}(k T) & \ddot{z}_{c}(k T) & f_{n}^{o}(k T)+M_{c} g
\end{array}\right]^{T}
$$


- The model constant matrices:

$$
\begin{aligned}
\boldsymbol{A} & =\left[\begin{array}{lllll}
1 & T & \frac{T^{2}}{2} & 0 & 0 \\
0 & 1 & T & 0 & 0 \\
0 & 0 & 1 & 0 & 0 \\
0 & 0 & 0 & 1 & T \\
0 & 0 & 0 & 0 & 1
\end{array}\right], \quad \boldsymbol{B}=\left[\begin{array}{ccc}
\frac{T^{3}}{6} & 0 \\
\frac{T^{2}}{2} & 0 \\
T & 0 \\
0 & \frac{T^{2}}{2} \\
0 & T
\end{array}\right] \\
\boldsymbol{C}_{z} & =\left[\begin{array}{ccccc}
1 & 0 & 0 & 0 & 0 \\
0 & 0 & 1 & 0 & 0 \\
0 & 0 & -M_{c} & 1 & 0
\end{array}\right]
\end{aligned}
$$

- $\boldsymbol{v}_{z}$ is the observation noise and considered as a Gaussian white noise with a covariance matrix $\boldsymbol{R}_{z}$. Thus, $\boldsymbol{v}_{z} \sim$ $N\left(\mathbf{0}, \boldsymbol{R}_{z}\right)$.

To formulate the estimation problem as a Kalman filter, we reformulate the dynamic system in (14) as follows:

$$
\begin{aligned}
\mathcal{Z}(k+1) & =\boldsymbol{A} \mathcal{Z}(k)+\boldsymbol{\omega}_{z}(k) \\
\boldsymbol{Y}_{z}(k) & =\boldsymbol{C}_{z} \mathcal{Z}(k)+\boldsymbol{v}_{z}(k)
\end{aligned}
$$

where $\boldsymbol{\omega}_{z}(k)=\boldsymbol{B} \boldsymbol{u}_{z}(k)$ becomes the process noise, which is supposed to be a Gaussian white noise with a covariance $\boldsymbol{Q}$, i.e. $\boldsymbol{\omega}_{z} \sim N(\mathbf{0}, \boldsymbol{Q})$, which is independent of $v_{z}$. The matrix $Q$ is given by:

$$
\boldsymbol{Q}=\boldsymbol{B}\left[\begin{array}{cc}
\sigma_{\ddot{z}_{c}}^{2} & 0 \\
0 & \sigma_{\ddot{F}_{z}}^{2}
\end{array}\right] \boldsymbol{B}^{T}
$$

where $\sigma_{\ddot{z}_{c}}^{2}$ and $\sigma_{\ddot{F}_{z}}^{2}$ are respectively the covariance of $\dddot{z}_{c}$ and $\ddot{F}_{z}$.

By applying Kalman filter on (16), an estimation of the state vector is obtained:

$$
\hat{\mathcal{Z}}(k)=\left[\begin{array}{lllll}
\hat{z}_{c}(k T) & \hat{\dot{z}}_{c}(k T) & \hat{\ddot{z}}_{c}(k T) & \hat{F}_{z}(k T) & \hat{\dot{F}}_{z}(k T)
\end{array}\right]^{T}
$$

\section{B. Estimating $F_{x}$ and $F_{y}$}

As above-mentioned $F_{x}$ and $F_{y}$ can be estimated from (7). However, because of the similarity between the two components of ZMP $\left(p_{x}\right.$ and $\left.p_{y}\right)$, we only consider the estimation of $F_{x}$ as $F_{y}$ can be estimated in an analogous way.

At first, $p_{x}$ in (7) is discretized with a sampling time $T$ to obtain the following linear dynamic system:

$$
\begin{aligned}
\mathcal{X}(k+1) & =\boldsymbol{A} \mathcal{X}(k)+\boldsymbol{\omega}_{x}(k) \\
\boldsymbol{Y}_{x}(k) & =\boldsymbol{C}_{x} \boldsymbol{\mathcal { X }}(k)+\boldsymbol{v}_{x}(k)
\end{aligned}
$$

where:

- $\mathcal{X}(k), \boldsymbol{\omega}_{x}(k), \boldsymbol{v}_{x}, \boldsymbol{A}$ and $\boldsymbol{Q}$ are defined in the same way as in Section IV-A by replacing $F_{z}$ by $F_{x}$ and $z_{c}$ by $x_{c}$.

- The output signal:

$$
\boldsymbol{Y}_{x}(k)=\left[\begin{array}{lll}
x_{c}(k T) & \ddot{x}_{c}(k T) & p_{x}(k T)
\end{array}\right]^{T}
$$

- The output matrix:

$$
\begin{aligned}
& \boldsymbol{C}_{x}=\left[\begin{array}{ccccc}
1 & 0 & 0 & 0 & 0 \\
0 & 0 & 1 & 0 & 0 \\
1 & 0 & \frac{M_{c} \hat{z}_{c}(k T)}{\hat{f}_{n}^{o}(k T)} & -\frac{\hat{z}_{c}(k T)}{\hat{f}_{n}^{o}(k T)} & 0
\end{array}\right] \\
& \hat{z}_{c}(k T) \text { and } \hat{f}_{n}^{o}(k T)=-M_{c} g-M_{c} \hat{\ddot{z}}_{c}(k T)+\hat{F}_{z}(k T) \\
& \text { are computed using (18). }
\end{aligned}
$$

By applying Kalman filter on (19), an estimation of the state vector $\mathcal{X}$ is obtained.

The observer implementation architecture is summarized in Fig. 4.

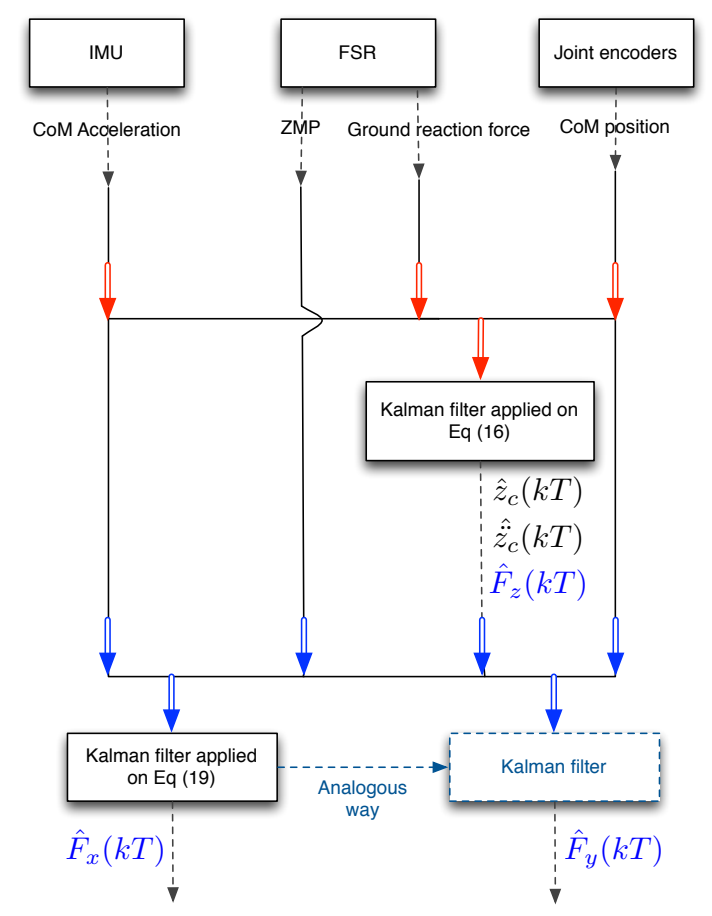

Fig. 4. Block diagram of the external force observer $\hat{\boldsymbol{F}}^{e x t}=\left[\begin{array}{lll}\hat{F}_{x} & \hat{F}_{y} & \hat{F}_{z}\end{array}\right]^{T}$

\section{Integration Into Walking Pattern Generation \\ VI. EXPERIMENTAL RESULTS}

For the reported experiments results, the following parameters were used: $g=9.81$ m.s ${ }^{-2}, T=16.7 \mathrm{~ms}(F=60 \mathrm{~Hz})$ and $M_{c}=5.19 \mathrm{~kg}$.

Regarding the observer parameters, the best results have been obtained with:

$$
\begin{aligned}
\sigma_{\ddot{x}}^{2} & =\sigma_{\ddot{y}}^{2}=\sigma_{\ddot{z}}^{2}=10^{3} \\
\sigma_{\ddot{F}_{x}}^{2} & =\sigma_{\ddot{F}_{y}}^{2}=\sigma_{\ddot{F}_{z}}^{2}=10^{3} \\
\boldsymbol{R}_{z} & =\left[\begin{array}{ccc}
0.01 & 0 & 0 \\
0 & 1 & 0 \\
0 & 0 & 1
\end{array}\right], \boldsymbol{R}_{x}=\boldsymbol{R}_{y}=\left[\begin{array}{ccc}
0.01 & 0 & 0 \\
0 & 1 & 0 \\
0 & 0 & 0.01
\end{array}\right]
\end{aligned}
$$

Moreover, the experimental data as well as $\mathrm{C}++/ \mathrm{ROS}$ implementation and the MATLAB code that has been used to produce the results in this paper can be downloaded here ${ }^{2}$.

${ }^{2}$ https://goo.gl/VkhejY 
These data and code could be helpful for the humanoid robotics community with the objective of benchmarking and comparing with other/their approaches.

The observer has been experimentally validated on the Nao robot through three scenarios, and it has been also used to accomplish human-robot interaction tasks.

\section{A. Force Applied to the Robot Torso}

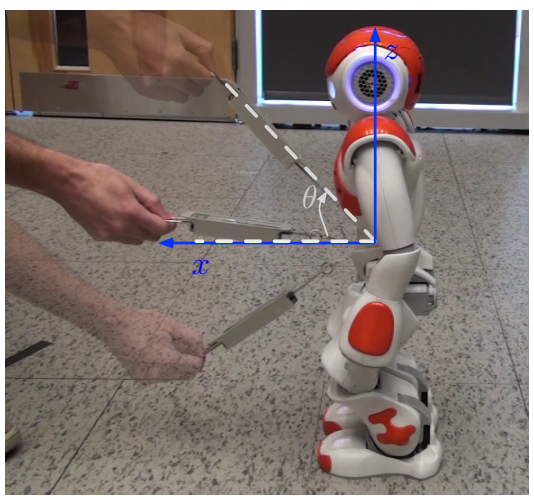

(a) Experimental setup

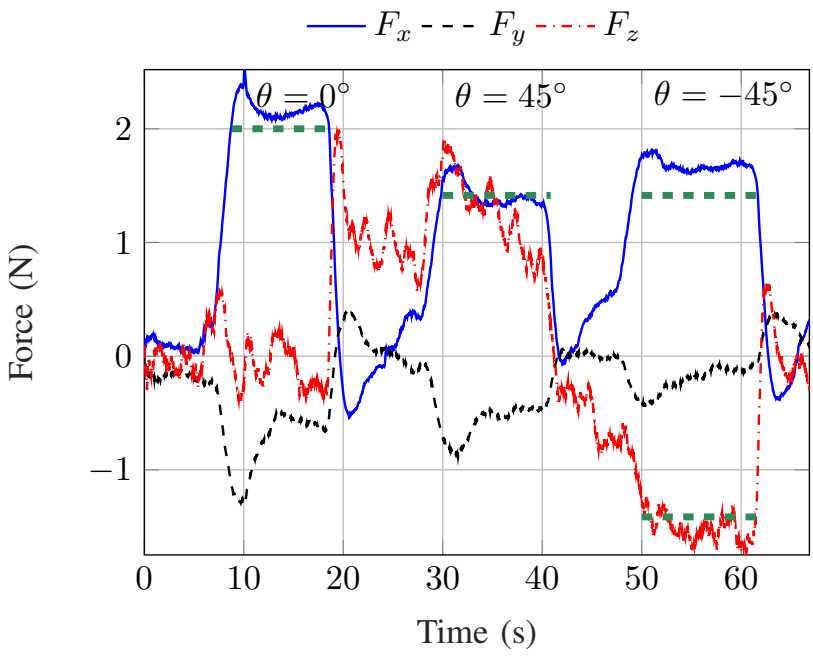

(b) Estimating the three components of $F_{e x t}$, the expected force is in green dashed line.

Fig. 5. Experiment 1: Applying a fixed-magnitude force in the $x-z$ plane.

In this experiment, a force in the $x-z$ plane is applied through a spring-based force meter as shown in Fig. 5(a). As the torso frame is close to the CoM position of the robot, the magnitude of resulting moment applied to the LIPM model is relatively small. Therefore, applying a force to the robot torso is practically equivalent to applying it to its CoM.

The $F_{x}$ and $F_{y}$ components of the force can be easily obtained by the following formula:

$$
\begin{gathered}
F_{x}=\left\|F^{e x t}\right\| \cos (\theta) \\
F_{z}=\left\|F^{e x t}\right\| \sin (\theta)
\end{gathered}
$$

where $\left\|F^{e x t}\right\|$ is the force magnitude measured with the force meter, and $\theta$ is the angle between the force meter and the $x$ axis, the positive direction of rotation is defined to be clockwise.

The experiment consists of applying a force with a constant magnitude of approximately $2 \mathrm{~N}$ in the $x-z$ plane but with different values of the angle $\theta$.

Fig. 5(b) shows the overall performance of the observer, it points out that the observer reasonably succeeds in estimating the three components of the external force. However, note that the main difficulty in this experiment was keeping a force with constant magnitude by only visually monitoring the force indicator. Moreover, the angle $(\theta)$ was also visually monitored using a protractor.

We can also notice that the observer detects non-zero force along $y$ axis, this is mainly because of the difficulty of keeping the force meter in the vertical plane.

\section{B. Applying a Force to a Point of the Robot Arm}

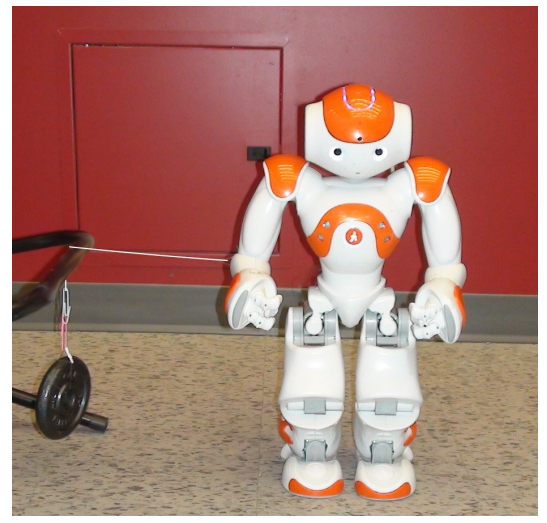

(a) Experimental setup

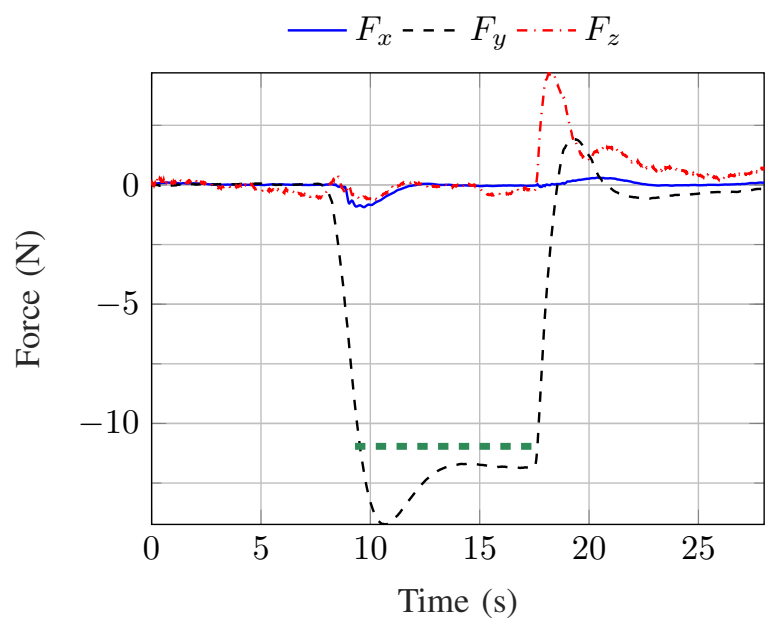

(b) Estimating the three components of $F_{\text {ext }}$, the expected force is in green dashed line.

Fig. 6. Experiment 2: Applying a force along $y$ axis

The setup for the second and third scenarios are respectively presented in Fig. 6(a) and Fig. 7(a), and the attached mass $m_{o}=2.5$ pounds $(1.13 \mathrm{~kg})$. In both experiments, a force of around $11 \mathrm{~N}$ has been applied to the robot right arm, first along the $y$ axis in Fig. 6(a) and then along the $z$ axis in Fig. 7(a). 


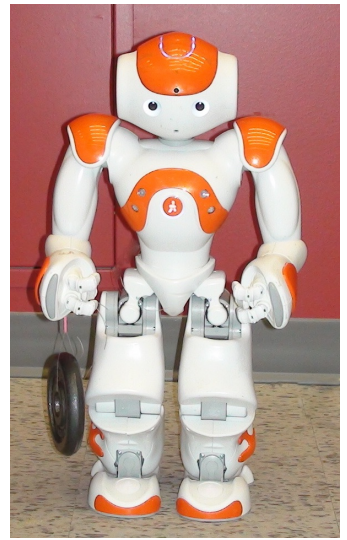

(a) Experimental setup

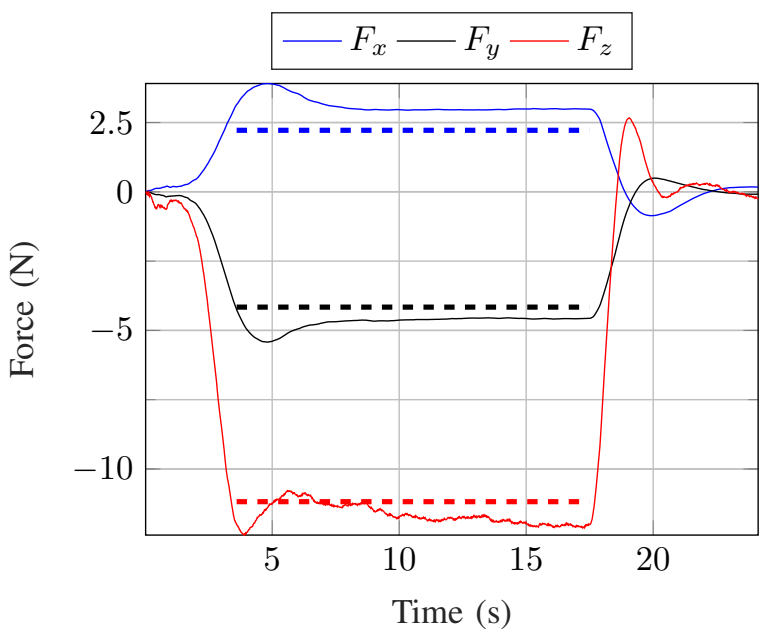

(b) Estimating the three components of $F_{\text {ext }}$ and its norm, the expected forces are in dashed lines

Fig. 7. Experiment 3: Applying an arbitrary external force

By applying inverse dynamics to the kinematic chain from the point at which the force is applied to the robot CoM, the wrench vectors in the second and third experiments as well as the virtual forces, as defined in (11), are respectively:

$$
\begin{aligned}
& \mathcal{W}_{2}=\left[\begin{array}{l}
\boldsymbol{F}_{2}^{\text {ext }} \\
\boldsymbol{\tau}_{2}^{\text {ext }}
\end{array}\right] \approx\left[\begin{array}{c}
0.0 \\
-11.12 \\
0.0 \\
-0.05 \\
0.0 \\
-0.08
\end{array}\right] \Longrightarrow \hat{\boldsymbol{F}}_{2}^{\text {ext }} \approx\left[\begin{array}{c}
0.0 \\
-10.96 \\
0
\end{array}\right] \\
& \mathcal{W}_{3}=\left[\begin{array}{l}
\boldsymbol{F}_{3}^{\text {ext }} \\
\boldsymbol{\tau}_{3}^{\text {ext }}
\end{array}\right] \approx\left[\begin{array}{c}
0.0 \\
0.0 \\
-11.12 \\
1.31 \\
0.70 \\
0.0
\end{array}\right] \Longrightarrow \hat{\boldsymbol{F}}_{3}^{\text {ext }} \approx\left[\begin{array}{c}
2.22 \\
-4.16 \\
-11.12
\end{array}\right]
\end{aligned}
$$

Those experiments are very similar to the experiments conducted in [13], they are used as a benchmark to compare with their results. Fig. 6(b) and Fig. 7(b) show that the proposed observer succeeds in estimating the corresponding virtual external force, this is because the estimated force is reasonably close to the expected one.

The authors in [13] point out that the estimated force, by their method, has an important magnitude instead of a small one when the mass is removed. They argue that their main sources of error are the unmodeled static friction and the lowcost hardware of Nao robot, for instance, plastic joint gears. On the other hand, the estimated force by our observer after removing the mass has a small magnitude of around $0.5 \mathrm{~N}$, which is an order of magnitude smaller than the results in [13]. Furthermore, those results also show the robustness of our observer to the sources of error identified in [13].

\section{Compliant Human-Robot Interaction}

As an application of our observer, we have implemented an interactive walking based on the interaction between the robot and a human as shown in Fig. 8. In this scenario, the robot walks in the direction of the detected force (forward or backward) if the force magnitude exceeds a predefined threshold, and stops if the magnitude of detected force is less than the threshold.
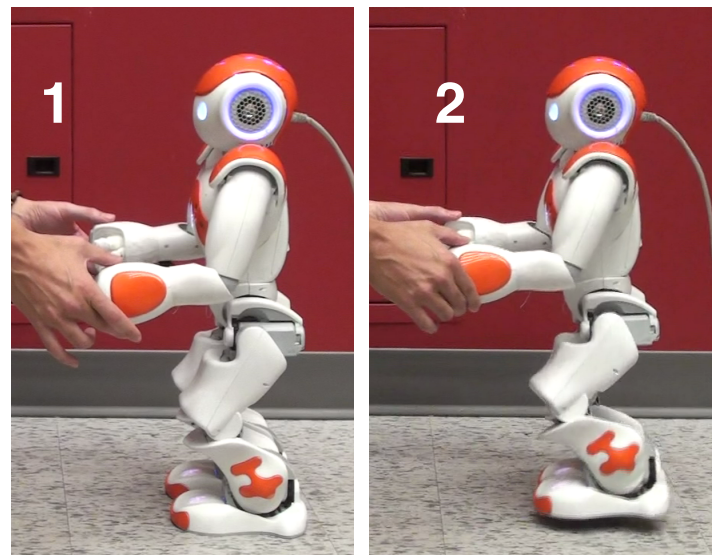

Fig. 8. Interactive walking by applying a sagittal guidance force to the robot hands
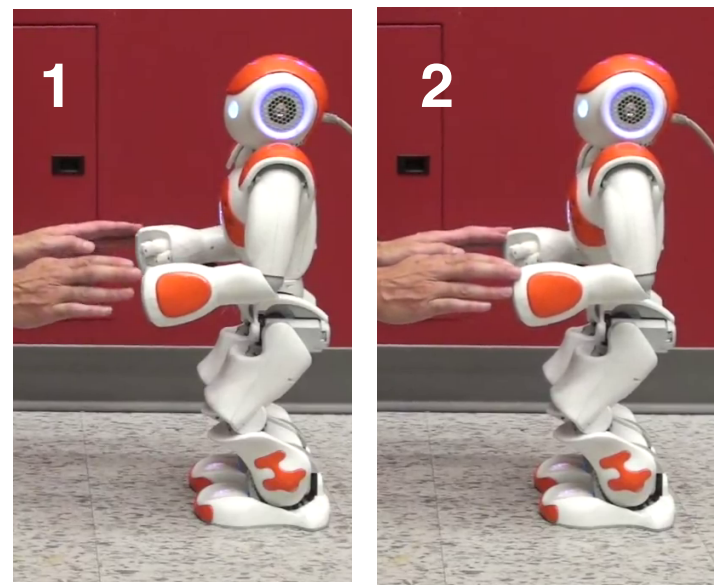

Fig. 9. Compliant control of the robot arms

A second application is a compliant control of the robot arms as a function of the detected force in the sagittal plane ( $x$ axis) as follows: 


$$
x_{h}=x_{h}^{0}+\frac{1}{k} F_{x}
$$

where $x_{h}$ and $x_{h}^{0}$ are respectively the desired position of the hand and its initial position along the $x$ axis. $k$ is a virtual spring constant and $F_{x}$ is the detected force along $x$ axis. A snapshot of the experiment is given in Fig. 9.

\section{CONCLUSION}

In this paper, we introduced a Kalman filter based observer to estimate the three components of an external force applied to a medium-sized/small humanoid robot. In contrast to conventional methods that are mainly based on the use of expensive 6-axis force/torque sensors, the proposed observer uses measurement from force-sensing resistors located under the feet of the robot and the information from the robot's IMU sensor. This observer was extensively tested on a Nao robot and the results revealed that it overcomes the limitations of the related approaches in the literature, those limitations are mainly caused by the low-cost hardware components of the robot.

However, there is still room for improvement, for instance, the covariance matrices $R$ and $Q$ in the observer implementation have been experimentally identified, however, they can be estimated using some existing methods in the literature, such as [18]. Moreover, even though the objective of our observer is to estimate an equivalent force, called virtual force, that is applied to the CoM frame, a combination with methods for identifying the contact point, such as [19] or [20], would provide the external force components at the contact point and in the world frame by a simple inverse dynamics calculation.

Future work will focus on integrating the external force observer into the pattern generator module and humanhumanoid robot collaborative tasks.

\section{ACKNOWLEDGMENT}

This research is supported by the Natural Sciences and Engineering Research Council of Canada (NSERC) under the grant RGPIN-2012-419406.

\section{REFERENCES}

[1] L. Hawley and W. Suleiman, "External Force Observer for Mediumsized Humanoid Robots," in IEEE-RAS International Conference on Humanoid Robots, 2016, pp. 366-371.

[2] A. Rioux and W. Suleiman, "Humanoid navigation and heavy load transportation in a cluttered environment," in IEEE/RSJ International Conference on Intelligent Robots and Systems (IROS), 2015, pp. 21802186.

[3] — " "Autonomous SLAM based humanoid navigation in a cluttered environment while transporting a heavy load," Robotics and Autonomous Systems, vol. 99, pp. 50-62, 2018.

[4] A. Rioux, C. Esteves, J.-B. Hayet, and W. Suleiman, "Cooperative SLAM-Based Object Transportation by Two Humanoid Robots in a Cluttered Environment," in IEEE-RAS International Conference on Humanoid Robots, 2015, pp. 331-337.

[5] — - "Cooperative Vision-Based Object Transportation by Two Humanoid Robots in a Cluttered Environment," International Journal of Humanoid Robotics, vol. 14, no. 03, pp. 1-30, 2017.
[6] K. Harada, S. Kajita, K. Kaneko, and H. Hirukawa, "ZMP Analysis for Arm / Leg Coordination," in IEEE/RSJ International Conference on Intelligent Robots and Systems, 2003, pp. 75-81.

[7] — , "Pushing manipulation by humanoid considering two-kinds of ZMPs," IEEE International Conference on Robotics and Automation, pp. 1627-1632, 2003.

[8] K. Harada, S. Kajita, H. Saito, M. Morisawa, F. Kanehiro, K. Fujiwara, K. Kaneko, and H. Hirukawa, "A humanoid robot carrying a heavy object," in IEEE International Conference on Robotics and Automation (ICRA), 2005, pp. 1712-1717.

[9] K. Kaneko, F. Kanehiro, M. Morisawa, E. Yoshida, and J.-P. Laumond, "Disturbance observer that estimates external force acting on humanoid robots," 12th IEEE International Workshop on Advanced Motion Control (AMC), pp. 1-6, 2012.

[10] M. Benallegue, P. Gergondet, H. Audren, A. Mifsud, M. Morisawa, F. Lamiraux, A. Kheddar, and F. Kanehiro, "Model-based external force/moment estimation for humanoid robots with no torque measurement," in IEEE International Conference on Robotics and Automation (ICRA), 2018, pp. $3122-3129$.

[11] K. Kaneko, F. Kanehiro, S. Kajita, M. Morisawa, K. Fujiwara, K. Harada, and H. Hirukawa, "Slip observer for walking on a low friction floor," in IEEE/RSJ International Conference on Intelligent Robots and Systems (IROS), 2005, pp. 1457-1463.

[12] D. Gouaillier, V. Hugel, P. Blazevic, C. Kilner, J. Monceaux, P. Lafourcade, B. Marnier, J. Serre, and B. Maisonnier, "Mechatronic design of NAO humanoid," IEEE International Conference on Robotics and Automation, pp. 769-774, 2009.

[13] T. Mattioli and M. Vendittelli, "Interaction Force Reconstruction for Humanoid Robots," IEEE Robotics and Automation Letters, vol. 2, no. 1, pp. 282-289, 2017.

[14] N. Rotella, A. Herzog, S. Schaal, and L. Righetti, "Humanoid Momentum Estimation Using Sensed Contact Wrenches," in IEEE-RAS International Conference on Humanoid Robots, 2015, pp. 556-563.

[15] E. Berger, D. Vogt, N. Haji-Ghassemi, B. Jung, and H. B. Amor, "Inferring guidance information in cooperative human-robot tasks," in IEEE- RAS International Conference on Humanoid Robots, 2015, pp. $124-129$.

[16] J. Carpentier, M. Benallegue, and N. Mansard, "Center-of-Mass Estimation for a Polyarticulated System in Contact - A Spectral Approach," IEEE Transactions on Robotics, vol. 32, no. 4, pp. 810822, 2016.

[17] M. Vukobratović and B. Borovac, "Zero-Moment Point - Thirty Five Years of Its Life," International Journal of Humanoid Robotics, vol. 01, no. 01, pp. 157-173, 2004.

[18] R. Mehra, "On the identification of variances and adaptive kalman filtering," IEEE Transactions on Automatic Control, vol. 15, no. 2, pp. 175-184, April 1970.

[19] F. Flacco and A. Kheddar, "Contact detection and physical interaction for low cost personal robots," in IEEE International Symposium on Robot and Human Interactive Communication (RO-MAN), 2017.

[20] F. Flacco, A. Paolillo, and A. Kheddar, "Residual-based contacts estimation for humanoid robots," in IEEE-RAS 16th International Conference on Humanoid Robots (Humanoids), Cancun, Mexico, Nov. 2016, pp. $409-415$. 